\title{
No short hair behaviors of ultra-compact stars
}

\author{
Yan Peng ${ }^{1,2, a}$ \\ ${ }^{1}$ School of Mathematical Sciences, Qufu Normal University, Qufu 273165, Shandong, China \\ ${ }^{2}$ Center for Gravitation and Cosmology, College of Physical Science and Technology, Yangzhou University, Yangzhou 225009, China
}

Received: 5 February 2021 / Accepted: 6 March 2021 / Published online: 21 March 2021

(C) The Author(s) 2021

\begin{abstract}
In the black hole spacetime, a no short hair theorem was proved, which states that the effective radius of black hole hairs must extend beyond the null circular orbit. In the present paper, in the horizonless gravity, we find a similar no short hair behavior that the effective radius of matter fields must also extend beyond the null circular orbit of ultra-compact stars. We should emphasize that the theorem presented in this manuscript is valid only for spatially regular matter configurations whose energy-momentum traces are non-negative.
\end{abstract}

\section{Introduction}

One of the most interesting predictions of general relativity is the existence of null circular orbits outside compact astrophysical objects, such as black holes and horizonless ultra-compact stars [1,2]. These null circular geodesics provide the way that massless particles (photons, gravitons) can orbit a central compact objects, which gives important information about the highly curved spacetime. At present, the null circular orbits have been widely studied

Recently, it was proved that the null circular orbit can be used to describe the distribution of exterior matter fields outside black holes. In spherically symmetric hairy black hole spacetimes, it was found that the effective radius of hairs must extend beyond the null circular orbit $[22,23]$. The authors in [22] have proposed a nice heuristic physical picture that the null circular orbit divides matter fields into two parts (fields below the orbit tending to be swallowed by black holes and fields above the orbit tending to be radiated away to infinity) and the self-interaction between these two parts bind together fields in different regions leading to the existence of black hole hairs. And it was further conjectured that the region above the null circular orbit contains at least half of the total hair mass [23-26].

a e-mail: yanpengphy@163.com (corresponding author)
The main goal of this paper is to study properties of horizonless ultra-compact spherical stars with null circular orbits. We shall analytically prove a no short hair theorem that the effective radius of matter fields must extend beyond the null circular orbit.

\section{Investigations on effective radius of matter fields}

We consider static horizonless stars with null circular orbits. In Schwarzschild coordinates, these ultra-compact spherically symmetric objects take the following form [22,23]

$d s^{2}=-g(r) e^{-2 \chi(r)} d t^{2}+\frac{d r^{2}}{g(r)}+r^{2}\left(d \theta^{2}+\sin ^{2} \theta d \phi^{2}\right)$.

The metric functions $\chi(r)$ and $g(r)$ only depend on the Schwarzschild radial coordinate r. Asymptotical flatness of the spacetime at the infinity requires that $g(r \rightarrow \infty)=1$ and $\chi(r \rightarrow \infty)=0$. Near the origin, regularity conditions of the gravity are $[27,28]$

$g(r \rightarrow 0)=1+O\left(r^{2}\right)$ and $\chi(0)<\infty$.

We take $\rho=-T_{t}^{t}, p=T_{r}^{r}$ and $p_{\tau}=T_{\theta}^{\theta}=T_{\phi}^{\phi}$, where $\rho, p$ and $p_{\tau}$ are interpreted as the energy density, the radial pressure and the tangential pressure respectively [23]. Einstein differential equations $G_{v}^{\mu}=8 \pi T_{v}^{\mu}$ yield the metric equations

$g^{\prime}=-8 \pi r \rho+\frac{1-g}{r}$,

$\chi^{\prime}=\frac{-4 \pi r(\rho+p)}{g}$.

We label $m(r)$ as the gravitational mass contained within a sphere of radial radius $r$. It can be expressed by the integral relation

$m(r)=\int_{0}^{r} 4 \pi r^{\prime 2} \rho\left(r^{\prime}\right) d r^{\prime}$. 
Considering relations (3) and (5), one can express the metric function $g(r)$ in the form [28]

$g=1-\frac{2 m(r)}{r}$.

Following approaches in [23], we obtain equations of null circular orbits. As the metric (1) is independent of the time $t$ and angular coordinates $\phi$, the geodesic trajectories are characterized by conserved energy $\mathrm{E}$ and conserved angular momentum L. And the null circular orbits are determined by an effective potential

$V_{r}=\left(1-e^{2 \chi}\right) E^{2}+g \frac{L^{2}}{r^{2}}$

with the characteristic relations

$V_{r}=E^{2}$ and $V_{r}^{\prime}=0$.

Substituting Einstein equations (3) and (4) into (7) and (8), we deduce the null circular orbit equation [27]

$N\left(r_{\gamma}\right)=3 g\left(r_{\gamma}\right)-1-8 \pi\left(r_{\gamma}\right)^{2} p\left(r_{\gamma}\right)=0$,

where we have introduced a new function $N(r)=3 g(r)-$ $1-8 \pi r^{2} p(r)$. The discrete roots of (9) correspond to the radii of the null circular orbit.

With the relation (2) and the regular condition $p(0)<\infty$, one obtains

$N(0)=2$.

We label $r_{\gamma}^{i n}$ as the radius of the innermost null circular orbit. That is to say $r_{\gamma}^{i n}$ is the smallest positive root of $N(r)=$ 0 . Also considering relation (10), one deduces that

$N(r) \geqslant 0$ for $r \in\left[0, r_{\gamma}^{i n}\right]$.

The conservation equation $T_{\nu ; \mu}^{\mu}=0$ has only one nontrivial component

$T_{r ; \mu}^{\mu}=0$.

Substituting Eqs. (3) and (4) into (12), one gets equation of the pressure

$p^{\prime}(r)=\frac{1}{2 r g}\left[\left(3 g-1-8 \pi r^{2} p\right)(\rho+p)+2 g T-8 g p\right]$

with $T=-\rho+p+2 p_{\tau}$ standing for the trace of the energy momentum tensor.

With a new pressure function $P(r)=r^{4} p$, the Eq. (13) can be expressed as

$P^{\prime}(r)=\frac{r}{2 g}[N(\rho+p)+2 g T]$,

where $N=3 g-1-8 \pi r^{2} p$.
The energy condition usually plays a central role in determining the spacetime geometry. We assume the dominant energy condition

$\rho \geqslant|p|, \quad\left|p_{\tau}\right| \geqslant 0$,

which means that the energy density $\rho$ is non-negative and bounds the pressures [22,23]. We also assume the nonnegative trace of the energy momentum tensor expressed as

$T=-\rho+p+2 p_{\tau} \geqslant 0$.

In this work, we take the non-negative trace conditions [2730]. A well known example for such horizonless configurations is the gravitating Einstein-Yang-Mills solitons, which are characterized by the identity $T=0$ [31]. In contrast, the usual non-positive trace condition was imposed in the black hole spacetime [22,23].

From (11) and (14-16), one deduces that

$P^{\prime}(r) \geqslant 0 \quad$ for $r \in\left[0, r_{\gamma}^{i n}\right]$.

We impose the condition that $\rho$ goes to zero faster than $r^{-4}[22,23]$. Also considering (15) and $P(r)=r^{4} p(r)$, there is the asymptotical behavior

$P(r \rightarrow \infty)=0$.

Near the origin, the pressure function $P(r)$ has the asymptotical behavior

$P(r \rightarrow 0)=0$.

Relations (18) and (19) imply that $|P(r)|$ must have a local maximum value at some extremum point $r_{0}$. We can define $r_{m}=r_{0}$ as the effective radii of matter fields [22,23]. According to (17), $P(r)$ is an increasing function of $r$ before it reaches the innermost null circular orbit. So the effective radii have a lower bound

$r_{m} \geqslant r_{\gamma}^{i n}$,

which is the same as the no short hair theorem of black holes [22,23].

\section{Conclusions}

We investigated distributions of matter fields in the background of horizonless ultra-compact spherically symmetric stars. We assumed the dominant energy condition and the non-negative trace condition. We defined an effective matter field radius at an extremum point where the pressure function $|P(r)|$ possesses a local maximum value. Using analytical methods, we obtained a lower bound on the effective matter field radius expressed as $r_{m} \geqslant r_{\gamma}^{i n}$ with $r_{m}$ as the effective matter field radius and $r_{\gamma}^{i n}$ corresponding to the innermost null circular orbit radius. So we found a no 
short hair behavior that the effective matter field radius must extend beyond the innermost null circular orbit. We also emphasized that the theorem presented in this manuscript is valid only for spatially regular matter configurations whose energy-momentum traces are non-negative $T \geqslant 0$.

Acknowledgements We would like to thank the anonymous referees for the constructive suggestions to improve the manuscript. This work was supported by the Shandong Provincial Natural Science Foundation of China under Grant no. ZR2018QA008. This work was also supported by a grant from Qufu Normal University of China under Grant no. xkjjc201906. This work was also supported by the Youth Innovations and Talents Project of Shandong Provincial Colleges and Universities (Grant no. 201909118).

Data Availability Statement This manuscript has no associated data or the data will not be deposited. [Authors' comment: This work is based on analytical studies. So there is no numerical data.]

Open Access This article is licensed under a Creative Commons Attribution 4.0 International License, which permits use, sharing, adaptation, distribution and reproduction in any medium or format, as long as you give appropriate credit to the original author(s) and the source, provide a link to the Creative Commons licence, and indicate if changes were made. The images or other third party material in this article are included in the article's Creative Commons licence, unless indicated otherwise in a credit line to the material. If material is not included in the article's Creative Commons licence and your intended use is not permitted by statutory regulation or exceeds the permitted use, you will need to obtain permission directly from the copyright holder. To view a copy of this licence, visit http://creativecomm ons.org/licenses/by/4.0/.

Funded by SCOAP ${ }^{3}$.

\section{References}

1. J.M. Bardeen, W.H. Press, S.A. Teukolsky, Rotating black holes: locally nonrotating frames, energy extraction, and scalar synchrotron radiation. Astrophys. J. 178, 347 (1972)

2. S. Chandrasekhar, The Mathematical Theory of Black Holes (Oxford University Press, New York, 1983)

3. C.J. Goebel, Comments on the "vibrations" of a black hole. Astrophys. J. 172, L95 (1972)

4. E. Teo, Spherical photon orbits around a Kerr black hole. Gen. Relativ. Gravit. 35, 1909 (2003)

5. Pedro V.P. Cunha, Carlos A.R. Herdeiro, Eugen Radu, Fundamental photon orbits: black hole shadows and spacetime instabilities. Phys. Rev. D 96(2), 024039 (2017)

6. Jai Grover, Alexander Wittig, Black hole shadows and invariant phase space structures. Phys. Rev. D 96(2), 024045 (2017)

7. Pedro V.P. Cunha, Carlos A.R. Herdeiro, Maria J. Rodriguez, Does the black hole shadow probe the event horizon geometry? Phys. Rev. D 97(8), 084020 (2018)

8. S.L. Shapiro, S.A. Teukolsky, Black Holes, White Dwarfs, and Neutron Stars: The Physics of Compact Objects (Wiley, New York, 1983)
9. V. Cardoso, A.S. Miranda, E. Berti, H. Witek, V.T. Zanchin, Geodesic stability, Lyapunov exponents and quasinormal modes. Phys. Rev. D 79, 064016 (2009)

10. S. Hod, Spherical null geodesics of rotating Kerr black holes. Phys. Lett. B 718, 1552 (2013)

11. Emanuel Gallo, J.R. Villanueva, Photon spheres in Einstein and Einstein-Gauss-Bonnet theories and circular null geodesics in axially-symmetric spacetimes. Phys. Rev. D 92(6), 064048 (2015)

12. Zdenek Stuchlik, Jan Schee, Bobir Toshmatov, Jan Hladik, Jan Novotny, Gravitational instability of polytropic spheres containing region of trapped null geodesics: a possible explanation of central supermassive black holes in galactic halos. JCAP 1706(06), 056 (2017)

13. Zdenek Stuchlik, Stanislav Hledik, Jan Novotny, General relativistic polytropes with a repulsive cosmological constant. Phys. Rev. D 94(10), 103513 (2016)

14. Ivan Zh Stefanov, Stoytcho S. Yazadjiev, Galin G. Gyulchev, Connection between black-hole quasinormal modes and lensing in the strong deflection limit. Phys. Rev. Lett. 104, 251103 (2010)

15. S. Hod, The fastest way to circle a black hole. Phys. Rev. D 84,104024 (2011)

16. Yan Peng, The extreme orbital period in scalar hairy Kerr black holes. Phys. Lett. B 792, 1-3 (2019)

17. S. Hod, Upper bound on the radii of black-hole photonspheres. Phys. Lett. B 727, 345 (2013)

18. P.V.P. Cunha, E. Berti, C.A.R. Herdeiro, Light-ring stability for ultracompact objects. Phys. Rev. Lett. 119, 251102 (2017)

19. Yan Peng, On instabilities of scalar hairy regular compact reflecting stars. JHEP 1810, 185 (2018)

20. Bahram Mashhoon, Stability of charged rotating black holes in the eikonal approximation. Phys. Rev. D 31(2), 290-293 (1985)

21. S. Hod, Universal bound on dynamical relaxation times and blackhole quasinormal ringing. Phys. Rev. D 75 (2007)

22. D. Núñez, H. Quevedo, D. Sudarsky, Black holes have no short hair. Phys. Rev. Lett. 76, 571 (1996)

23. S. Hod, hairy black holes and null circular geodesics. Phys. Rev. D 84, 124030 (2011)

24. Y.S. Myung, T. Moon, Hairy mass bound in the Einstein-BornInfeld black hole. Phys. Rev. D 86, 084047 (2012)

25. Y. Peng, Hair mass bound in the black hole with nonzero cosmological constants. Phys. Rev. D 98, 104041 (2018)

26. Y. Peng, Hair distributions in noncommutative Einstein-BornInfeld black holes. Nucl. Phys. B 941, 1-10 (2019)

27. S. Hod, Lower bound on the compactness of isotropic ultracompact objects. Phys. Rev. D 97, 084018 (2018)

28. S. Hod, Self-gravitating field configurations: the role of the energymomentum trace. Phys. Lett. B 739, 383 (2014)

29. J. Novotný, J. Hladík, Z. Stuchlík, Polytropic spheres containing regions of trapped null geodesics. Phys. Rev. D 95, 043009 (2017)

30. S. Hod, Analytic study of self-gravitating polytropic spheres with light rings. Eur. Phys. J. C 78, 417 (2018)

31. R. Bartnik, J. McKinnon, Particlelike solutions of the EinsteinYang-Mills equations. Phys. Rev. Lett. 61, 141 (1998) 